\title{
Self Tagging Studies Using Semileptonic B Decavs
}

\author{
A. B. Wicklund and K. Byrum \\ High Energy Physics Division \\ Argonne National Laboratory, Argonne, Ilinois 60439
}

16 March 1994

\section{1) Expectations}

We examine $B^{\circ}$ and $B^{+}$decays for evidence for "self-tagging". If isospin $1 / 2$ dominates the $\mathrm{b} \rightarrow \mathrm{B}$ or $\mathrm{B}^{* *} \rightarrow \mathrm{B}$ fragmentation processes, then we expect the correlations

$$
\begin{aligned}
& \mathrm{B}^{\circ} \pi^{+}>>\mathrm{B}^{\circ} \pi^{-} \\
& \mathrm{B}^{+} \pi^{-} \gg \mathrm{B}^{+} \pi^{+}
\end{aligned}
$$

We use the semileptonic decay modes

1) $\mathrm{B}^{+} \rightarrow \mathrm{e}^{+} v \quad \overline{\mathrm{D}}^{\circ}(*)$

$$
\overline{\mathrm{D}}^{\circ} \rightarrow \mathrm{K}^{+} \pi^{-}
$$

2) $\quad \mathrm{B}^{\circ} \rightarrow \mathrm{e}^{+} \mathrm{v} \quad \mathrm{D}^{-\left(^{*}\right)}$

$$
\mathrm{D}^{-} \rightarrow \mathrm{K}^{+} \pi^{-} \pi
$$

3) $\quad \mathrm{B}^{\circ} \rightarrow \mathrm{e}^{+} v \quad \mathrm{D}^{*-}$

$$
\begin{aligned}
\mathrm{D}^{*-} \rightarrow & \overline{\mathrm{D}}^{\circ} \pi_{\mathrm{b}} \\
& \overline{\mathrm{D}}^{\circ} \rightarrow \mathrm{K}^{+} \pi^{-} \\
& \mathrm{K}^{+} \pi^{-} \pi^{\circ}
\end{aligned}
$$

and their conjugates.

In sample (1) we exclude $\bar{D}^{\circ}$ 's that are accompanied by a bachelor pion candidate of either charge (RS or WS), satisfying the mass-difference cut 


$$
\mathrm{M}\left(\mathrm{K}^{+} \pi^{-} \pi_{\mathrm{b}}\right)-\mathrm{M}\left(\mathrm{K}^{+} \pi^{-}\right)<0.148 \mathrm{GeV}
$$

Thus, some $\mathrm{B}^{\circ} \mathrm{s}$ can leak into sample (1) if the bachelor pion is not reconstructed. Since most $D^{\circ} s$ come from $D^{* \circ} \rightarrow D^{\circ} \pi^{\circ}$, and since the bachelor reconstruction efficiency is demonstrably high, this is a small effect. Numerically, using recent CLEO data on the semileptonic $B R^{\prime}$ s into $D, D^{*}, D^{* *}$, we estimate that the total fraction of $D^{*+} \rightarrow D^{\circ} \pi^{+} b$ in the $D^{\circ}$ sample should be

$$
\mathrm{D}^{*+} \rightarrow \mathrm{D}^{\circ} \pi^{+}{ }_{\mathrm{b}} / \mathrm{All} \mathrm{D}^{\circ}=0.32 \pm .03
$$

where the error reflects (complete) uncertainty in the $D^{* *} B R s, D^{* *} \rightarrow D \pi$ versus $D^{*} \pi$. From the fitted $D^{\circ}$ and $D^{*+}$ peaks in the data, this observed ratio is $0.27 \pm .05$, implying that the efficiency for finding the bachelor pion is $85 \pm 15 \%$. A $15 \%$ inefficiency for finding the bachelor pion corresponds to $5 \%$ feed down from the $\mathrm{B}^{\circ}$ to the $\mathrm{B}^{+}$sample, i.e. negligible.

The $\mathrm{D}^{-}$in channel (2) cannot come from $\mathrm{D}^{* \circ}$ decays, only from direct (pseudoscalar) or $\mathrm{D}^{*-}$ production. Thus, $\mathrm{B}^{+} \mathrm{s}$ do not leak into channels (2) or (3), provided that only $\mathrm{J}^{\mathrm{p}}=0^{-}$and $1^{-}$states are produced in semileptonic decays.

$\mathrm{D}^{* *}$ states can cause cross talk between $\mathrm{B}^{\circ}$ and $\mathrm{B}^{+}$; for example,

$$
\begin{array}{ll}
\mathrm{B}^{+} \rightarrow \mathrm{e}^{+} \vee & \mathrm{D}^{* * 0}, \\
& \mathrm{D}^{* * \circ} \rightarrow \mathrm{D}^{*-} \pi^{+}
\end{array}
$$

can cause $\mathrm{B}^{+}$to be misidentified as $\mathrm{B}^{\circ}$ in channel (3). This would in general lead to a dilution in the self-tagging efficiency. However, if the pion from the $\mathrm{D}^{* *}$ decay is itself used as a tag (instead of other charged particles in the fragmentation), then we will get an enhancement in the apparent tagging rate, since these pions will exhibit exactly the same isospin correlations as the "self-tagging" signal. Numerically, taking "reasonable" values for the $D^{* *}$ fraction and the $D^{* *} \rightarrow D \pi, D^{*} \pi B R$ 's, we estimate that the different samples would have the following cross-talk from $D^{* *}$ 
decays: $14 \%\left(\mathrm{~B}^{+} \rightarrow \overline{\mathrm{D}}^{\circ}\right), 10 \%\left(\mathrm{~B}^{\circ} \rightarrow \mathrm{D}^{*-}\right)$, and $18 \%\left(\mathrm{~B}^{\circ} \rightarrow \mathrm{D}^{-}\right)$. In general, some fraction of these events would be (incorrectly) interpreted as right-sign tags, depending on how many charged tracks accompany the charged pion from the $D^{* *}$ decay.

\section{2) Results}

We summarize the results. Figures 1 and 2 show the $\mathrm{K}^{+} \pi$ mass spectra for channel (1). In Fig. (2) we define the highest Pt particle, in a cone of $R<1.0$, as the tag particle, and separate these into "same-sign" (SS) and "opposite-sign" (OS), with respect to the electron charge. Thus, the expected $B^{+} \pi$ isospin correlation should favor OS tags over SS tags. The fits give the following $\overline{\mathrm{D}}^{\circ}$ signal rates:

$\begin{array}{lll}\text { All } & \overline{\mathrm{D}}^{\circ} & 370 \pm 38 \\ \text { SS } & \overline{\mathrm{D}}^{\circ} & 142 \pm 25 \\ \text { OS } & \overline{\mathrm{D}}^{\circ} & 217 \pm 29 \\ & & \\ \text { SS } & \text { SB } & 1220 \pm 35 \\ \text { OS } & \text { SB } & 1768 \pm 42, \quad \text { "SB" = sidebands, } .04<\mathrm{dM}<.12 \\ & & \\ \rightarrow & \mathrm{D}\left(\mathrm{D}^{\circ}\right) & 0.21 \pm 0.11 \\ & \mathrm{D}(\mathrm{SB}) & 0.18 \pm 0.02,\end{array}$

where the dilution factor is defined as

$$
\mathrm{D}=(\mathrm{OS}-\mathrm{SS}) /(\mathrm{OS}+\mathrm{SS})
$$

If we just count events in the signal region $(a b s(d M)<.04)$ and compare with the sidebands (linear subtraction), then we get $\mathrm{D}$ (signal region) $=0.40 \pm 0.14$. This is consistent with the fit result within errors. We note that the charge correlation is essentially the same for the signal and the sidebands. That is not a cause for concern, since in principle we care only about the signal response. The reason why the sidebands exhibit a strong OS correlation is obvious -- the basic signal is $\mathrm{e}^{+} \mathrm{K}^{+} \pi$, 
so elementary charge conservation would require an excess of $\pi^{-}$over $\pi^{+}$tags, Indeed, any evidence for "self-tagging" based on $\mathrm{B}^{+}$alone (e.g. $\psi \mathrm{K}^{+}$for instance) cannot be convincing, that is, we cannot differentiate isospin from charge conservation.

Turning to the $\mathrm{B}^{\circ}$ decays, Figs. (3) - (6) show the $\mathrm{D}^{-} \rightarrow \mathrm{K}^{+} \pi-\pi$ signals. In all cases, we require that the three charged tracks register in the SVX and meet at a common vertex, using a simple $\chi^{2}$ criterion; we also require that the vertex be displaced by

$$
\operatorname{Lxy}\left(\mathrm{K}^{+} \pi \pi\right)>500 \mu \mathrm{M}
$$

Figures 3 and 4 are for $\mathrm{Pt}\left(\mathrm{D}^{+}\right)>3 \mathrm{GeV} / \mathrm{c}$, and Figs. 5 and 6 for $\mathrm{Pt}\left(\mathrm{D}^{+}\right)>5 \mathrm{GeV} / \mathrm{c}$. Figures 4 and 6 show the SS and OS signals, defined in the same way as for the $\mathrm{D}^{\circ}$ peak above. The fitted peaks yield:

$\begin{array}{llrr} & & \mathrm{P}_{\mathrm{t}}>3 & \\ \text { All } & \mathrm{D}^{-} & 132 \pm 22 & 111 \pm 19 \\ \text { SS } & \mathrm{D}^{-} & 81 \pm 17 & 64 \pm 14 \\ \text { OS } & \mathrm{D}^{-} & 55 \pm 17 & 49 \pm 14 \\ \text { SS } & \text { SB } & 532 \pm 23 & 300 \pm 17 \\ \text { OS } & \text { SB } & 627 \pm 25 & 318 \pm 18 \\ & & & \\ \rightarrow & \mathrm{D}\left(\mathrm{D}^{-}\right) & -0.19 \pm 0.18 & \\ & \mathrm{D}(\mathrm{SB}) & +0.08 \pm 0.03 & \end{array}$

Here the signal favors SS over OS, as expected for $\mathrm{B}^{\circ}$ decays, while the $\mathrm{SB}$ exhibits a preference for OS over SS, similar to the sidebands in $\mathrm{B}^{+} \rightarrow \mathrm{e}^{+} v \overline{\mathrm{D}}^{\circ}$. However, statistics are meager in this difficult mode. 
Finally, we consider channel $(3), B^{\circ} \rightarrow e^{+} \vee D^{*-}$. We combine two cleanly identified sources of $\mathrm{D}^{*-}$ :

1) $\mathrm{D}^{*-} \rightarrow \overline{\mathrm{D}}^{\circ} \pi^{-}, \overline{\mathrm{D}}^{\circ} \rightarrow \mathrm{K}^{+} \pi$, where we make no SVX requirement, but we make tight quality cuts on the electron to improve the signal. We cut on $\pm 45 \mathrm{MeV}$ for $[\mathrm{M}(\mathrm{K} \pi)-1865]$. This selection gives 1162 events with mass difference,

$$
\mathrm{dM}=\mathrm{M}\left(\mathrm{K} \pi \pi_{\mathrm{b}}\right)-\mathrm{M}(\mathrm{K} \pi),
$$

$\mathrm{dM}<0.22 \mathrm{GeV}$, and a $\mathrm{D}^{*}$ signal of about 140 events over background. For wrong-sign (WS) bachelors, the corresponding rates are 848 (total) and 0 (signal).

2) $\quad \mathrm{D}^{*-} \rightarrow \overline{\mathrm{D}}^{\circ} \pi^{-}, \overline{\mathrm{D}}^{\circ} \rightarrow \mathrm{K}^{+} \pi \mathrm{X}$

Here we rely on SVX to get a $\mathrm{D}^{*}$ signal. First, we require both $\mathrm{K}^{+}$and $\pi^{-}$tracks to have SVX links and satisfy

a) $\quad 0.05<\operatorname{Lxy}(\mathrm{K} \pi)<1.0 \mathrm{~cm}$ ("basic cut").

In addition we require that the electron have an SVX link, and we reconstruct the $\mathrm{B}^{\circ} \rightarrow \mathrm{e}^{+} \vee \overline{\mathrm{D}}^{\circ}$ decay vertex by pointing the $\mathrm{K}^{-} \pi^{+}$pair back to the electron track. Note that the bachelor pion is not included in this fit, but it must point directly along the $\mathrm{D}^{\circ}$ flight path, by kinematics (due to the low $\mathrm{Q}$ value in $\mathrm{D}^{*-} \rightarrow \mathrm{D}^{\circ} \pi^{-}$). However, the (possible) missing $\pi^{\circ}$ in the $\mathrm{D}^{\circ}$ decay does smear out the $\mathrm{D}^{\circ}$ direction, causing some smearing in the $\mathrm{B}$ decay vertex. To minimize this, we point the electron $+\left(\mathrm{K}^{-} \pi^{+}\right)$pair back to the primary vertex, treating it as the total $B$ meson 4-vector, and require that this " $B$ " intersect the primary vertex. Specifically, we cut on: 
b) $\quad$ abs $\left(\operatorname{Dvx}\left(" \mathrm{~B}^{\prime \prime}\right)\right)<.06 \mathrm{~cm}$.

Lxy ("B") > $0 \mathrm{~cm}$

$-0.1<[\operatorname{Lxy}(\mathrm{K} \pi)-\operatorname{Lxy}(" \mathrm{~B} ")]<1.0 \mathrm{~cm}$

("B cuts").

Figure (7a) shows the mass difference distribution after applying these "B cuts" to an inclusive $\mathrm{D}^{*}$ candidate sample, satisfying "tight" electron cuts; the shaded inset shows the WS bachelor spectrum, scaled by a factor 1.2 to agree in the high side tail region. Figure (7b) shows the same distributions, for events that pass the "basic" SVX cuts but fail the "B cuts". The "B cuts" eliminate $60 \%$ of the events, but they cost little if any signal; using the cleaner $\mathrm{D}^{\circ} \rightarrow \mathrm{K}^{-} \pi^{+}$exclusive events, we estimate that the "B cuts" preserve about $86 \%$ of the $D^{*}$ signal. Figure (8) shows the mass difference distributions for all "exclusive" $D^{*-} \rightarrow \bar{D}^{\circ} \pi^{-}, \bar{D}^{c} \rightarrow K^{+} \pi$, candidates (bottom), and after both the "basic" and "B cuts" (top). The "basic" SVX requirements reduce the signal in this case (that is why we retain non-SVX events in sample (a)), but the "B cuts" clean up the high side BG a lot, just as in the inclusive $\mathrm{D}^{*}$ decay case. Figure (9) shows the $\left(\mathrm{K}^{-} \pi^{+}\right)$mass spectra (WS bachelors shaded) for $\mathrm{D}^{*}$ candidates after "basic SVX" and "B cuts", and after a mass difference cut, $\mathrm{dM}<0.157 \mathrm{GeV}$. The $\mathrm{D}^{\circ} \rightarrow \mathrm{K}^{-} \pi^{+}$and $\mathrm{K}^{-} \pi^{+} \pi^{\circ}$ peaks are evident, and even before any BG subtraction, the "mass gap" between these two decays is very clean.

We combine samples (1) and (2) for tagging studies, where sample (2) is required to pass the "B cuts". We also require $\mathrm{M}\left(\mathrm{K}^{-} \pi^{+}\right)>1.4 \mathrm{GeV}$ for sample (2). This gives a total of about $575 \mathrm{D}^{*}$ candidates in sample (2), satisfying $\mathrm{dM}<0.157$ $\mathrm{GeV}$; after applying "tight electron cuts", this number comes down to 409. For the tagging analysis, the $\mathrm{D}^{*}$ signal is clean enough, for the "B cuts" sample (2), that we do not make tight electron cuts on this sample (we have to subtract BG's in any case). Finally, we use the (RS bachelor) spectrum of Fig. (7b), events that fail "B cuts", to define the BG shape in the mass difference plot. We could have used the 
"WS" bachelor spectrum, but this does not appear to describe the BG at small dM very well. Our choice reduces the overall $\mathrm{D}^{*}$ signal, but is in practice more conservative for measuring tagging rates.

Combining samples (1) and (2), Fig. (10) shows the mass-difference distributions for events with SS and OS tags. The shaded insets are the assumed BG shapes derived above. We see that the $B G$ shapes match the data very well above the $\mathrm{D}^{*}$ signal region. Figure 11 shows the same distributions, this time for WS bachelors; the points are all WS events passing "B cuts", the histograms are BG shapes derived from WS bachelors that fail "B cuts".

We obtain the following $D^{*}$ rates, after BG subtraction:

$\begin{array}{llr}\text { SS } & \mathrm{D}^{*} & 209 \pm 19 \\ \text { OS } & \mathrm{D}^{*} & 104 \pm 17 \\ \text { SS } & \mathrm{BG} & 1010 \pm 32 \\ \text { OS } & \text { BG } & 1214 \pm 35 \\ & & \\ \rightarrow & \mathrm{D}\left(\mathrm{D}^{*}\right)=-0.34 \pm 0.08 \\ & \mathrm{D}(\mathrm{BG})=+0.09 \pm 0.02\end{array}$

Here the "BG" is defined as the event rate for $0.157<\mathrm{dM}<0.220$. As with $\mathrm{D}^{+} \rightarrow \mathrm{K}^{-}$ $\pi^{+} \pi^{+}$, the BG prefers OS tag pions, while the signal obeys the isospin (SS) expectation.

Can we tell whether the tag pions come from $\mathrm{D}^{* *}$ decay or from the primary vertex? We find nothing very distinctive in the momentum spectra or in the invariant mass between the $\mathrm{D}^{*}$ and the tag pion (all distributions look similar, for signal and $\mathrm{BG}, \mathrm{RS}$ and WS bachelors). We expect the $\mathrm{D}^{* *}$ decay pions to come from the $\mathrm{B}^{-}$ decay point in the SVX, while the desired tag pions should come from the primary vertex. Recall that for the $D^{*}$ decay modes in sample (2), we have required that the $\mathrm{K}^{-} \pi^{+}$vertex be well separated from the primary (Lxy > $\left.500 \mathrm{muM}\right)$, so we can, in 
principle, detect whether the tags come from the primary or secondary vertices. Figure (12) shows the impact distribution for RS $D^{*}$ candidates $(\mathrm{dM}<.157 \mathrm{GeV}$ ), for both SS and OS tags (there is little difference); we plot Dvx with respect to the primary vertex (bottom) and the $\mathrm{B}^{-}$decay vertex (top). To make this plot, we have further required that the bachelor pion intersect the electron $-\left(\mathrm{K}^{-} \pi^{+}\right)$vertex, and we have fitted the $B$ vertex with the bachelor pion included. We require a good vertex $\chi^{2}$ for the combined fit (in defining "B cuts" above, we did not require the bachelor pion to meet the B decay vertex, as there is some efficiency loss in this requirement). It is clear that the tag pions come from the primary vertex, not the B decay vertex. For comparison, we make the same plot for the RS bachelor pions themselves (Fig. 10). Here the Dvx distribution is much better centered on the decay vertex (top) than the production vertex (bottom). To show that these plots are not biased, we compare the rms's for the Dvx distributions for RS and WS bachelors with the primary and B-decay vertices, after fitting the B decay vertex with the bachelor pions:

$\begin{array}{lll}\frac{\mathrm{RS}}{201 \mu \mathrm{M}} & \frac{\mathrm{WS}}{126 \mu \mathrm{M}} & \text { - primary vertex } \\ 104 \mu \mathrm{M} & 191 \mu \mathrm{M} & \text { - B decay vertex }\end{array}$

Based on these rms values, we conclude that the WS bachelors generally come from the primary vertex, even though our vertex fit requires that they also match the Bdecay vertex; the RS bachelors generally come from the B-decay vertex, as expected, and not the primary. Although we cannot exclude some $\mathrm{D}^{* *}$ contribution to the tag sample, given the large SS/OS tag ratio for $\mathrm{D}^{*}$ 's, and the clear association of most tags (unlike bachelor pions) with the primary vertex, we conclude that fake tags from $D^{* *}$ decays are not a big effect.

We summarize the dilution factors for signals and BGs as follows: 
$\mathrm{D}$ (signal)

$-0.34 \pm .08$

$-0.19 \pm .18$

$0.21 \pm .11$

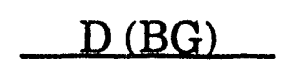

$+0.09 \pm .02$

$+0.08 \pm .03$

$+0.18 \pm .02$
Process

$\mathrm{B}^{\circ} \rightarrow \mathrm{e}^{+} \vee \mathrm{D}^{*-}$

$\mathrm{B}^{\circ} \rightarrow \mathrm{e}^{+} v \mathrm{D}^{-(*)}$

$\mathrm{B}^{+} \rightarrow \mathrm{e}^{+} \vee \overline{\mathrm{D}}^{\circ}(*)$

While the errors are large, they indicate that the signals, unlike the BG's, satisfy the isospin correlation. We can take the combined $\mathrm{B}^{\circ}$ self tag rates to be:

$$
\mathrm{D}\left(\mathrm{B}^{\circ}\right)=-.32 \pm .07 \text { (combined), }
$$

with a tagging efficiency close to $100 \%$. The $\mathrm{B}^{\circ}$ and $\mathrm{B}^{+}$correlations are consistent in magnitude. Assuming that they are equal, we obtain an average dilution factor

$$
\mathrm{D}\left(\mathrm{B}^{\circ}, \mathrm{B}^{+}\right)=\frac{\mathrm{RS}-\mathrm{WS}}{\mathrm{RS}+\mathrm{WS}}=+0.29 \pm .06
$$

\section{DISCLAIMER}

This report was prepared as an account of work sponsored by an agency of the United States Government. Neither the United States Government nor any agency thereof, nor any of their employees, makes any warranty, express or implied, or assumes any legal liability or responsibility for the accuracy, completeness, or usefulness of any information, apparatus, product, or process disclosed, or represents that its use would not infringe privately owned rights. Reference herein to any specific commercial product, process, or service by trade name, trademark, manufacturer, or otherwise does not necessarily constitute or imply its endorsement, recommendation, or favoring by the United States Government or any agency thereof. The views and opinions of authors expressed herein do not necessarily state or re.lect those of the United States Government or any agency thereof.

* Work supported in part by the U.S. Department of Energy, Division of High Energy Physics, Contract W-31-109-ENG-38. 


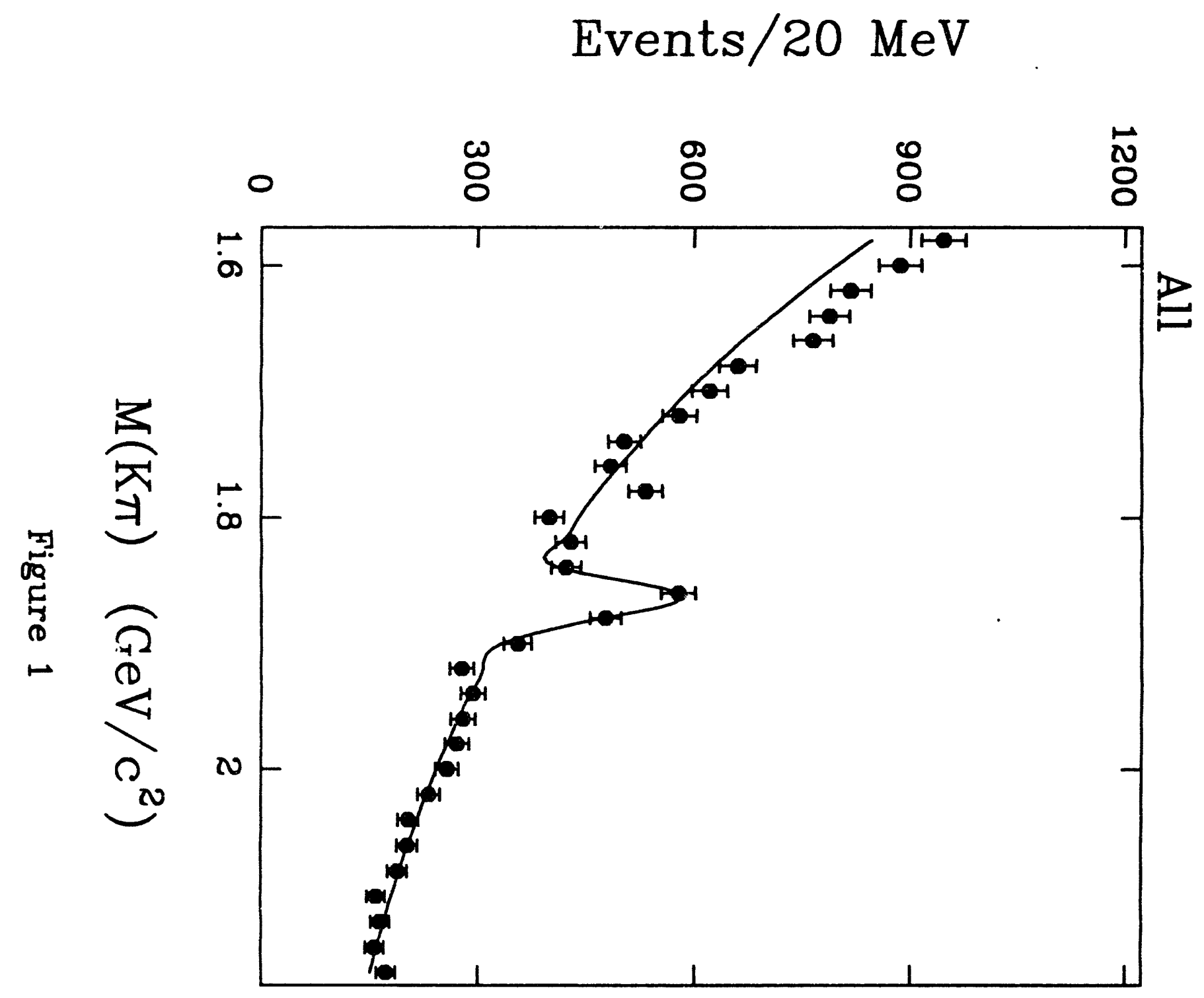




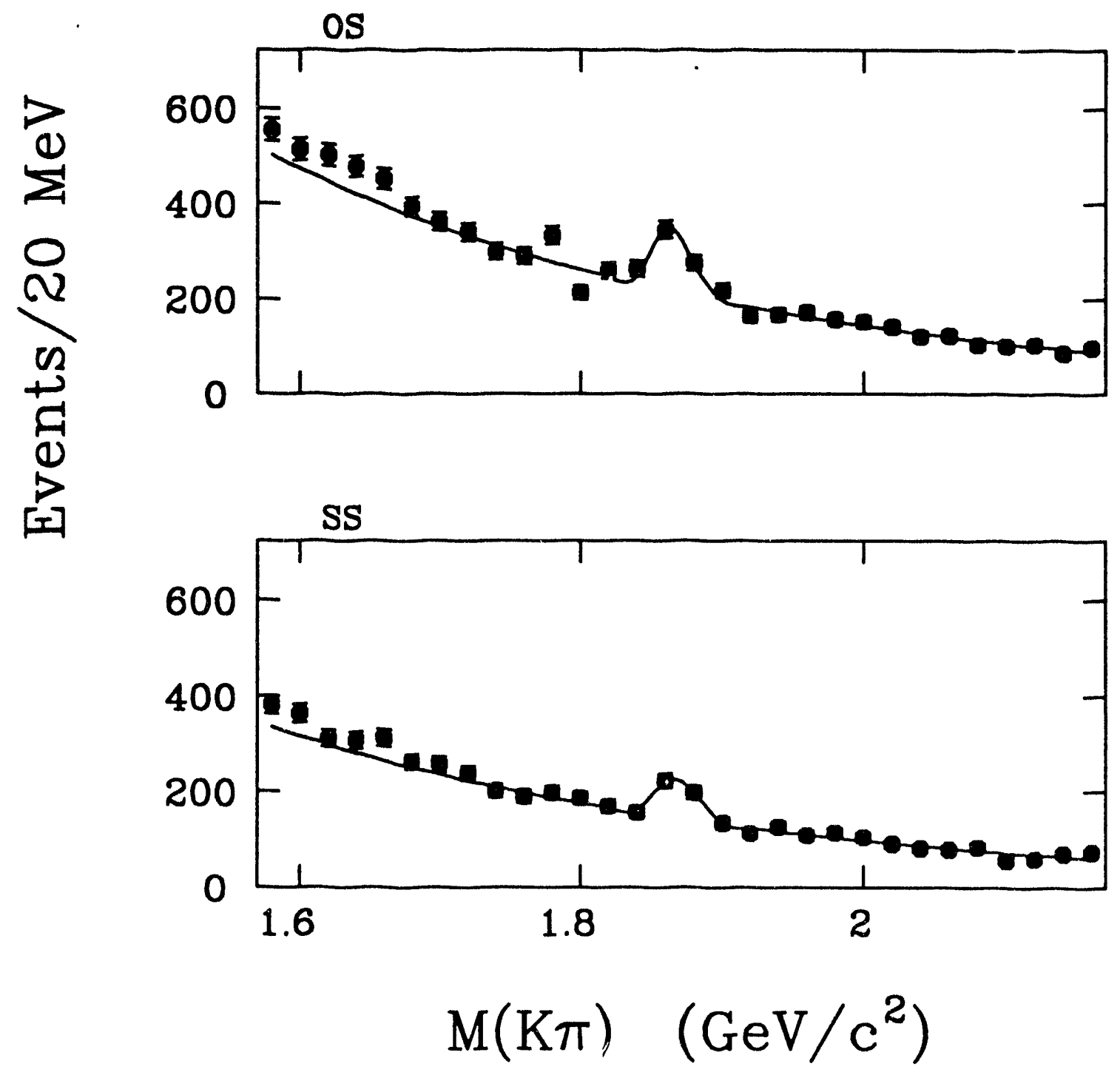

Figure 2 
Events/15 MeV

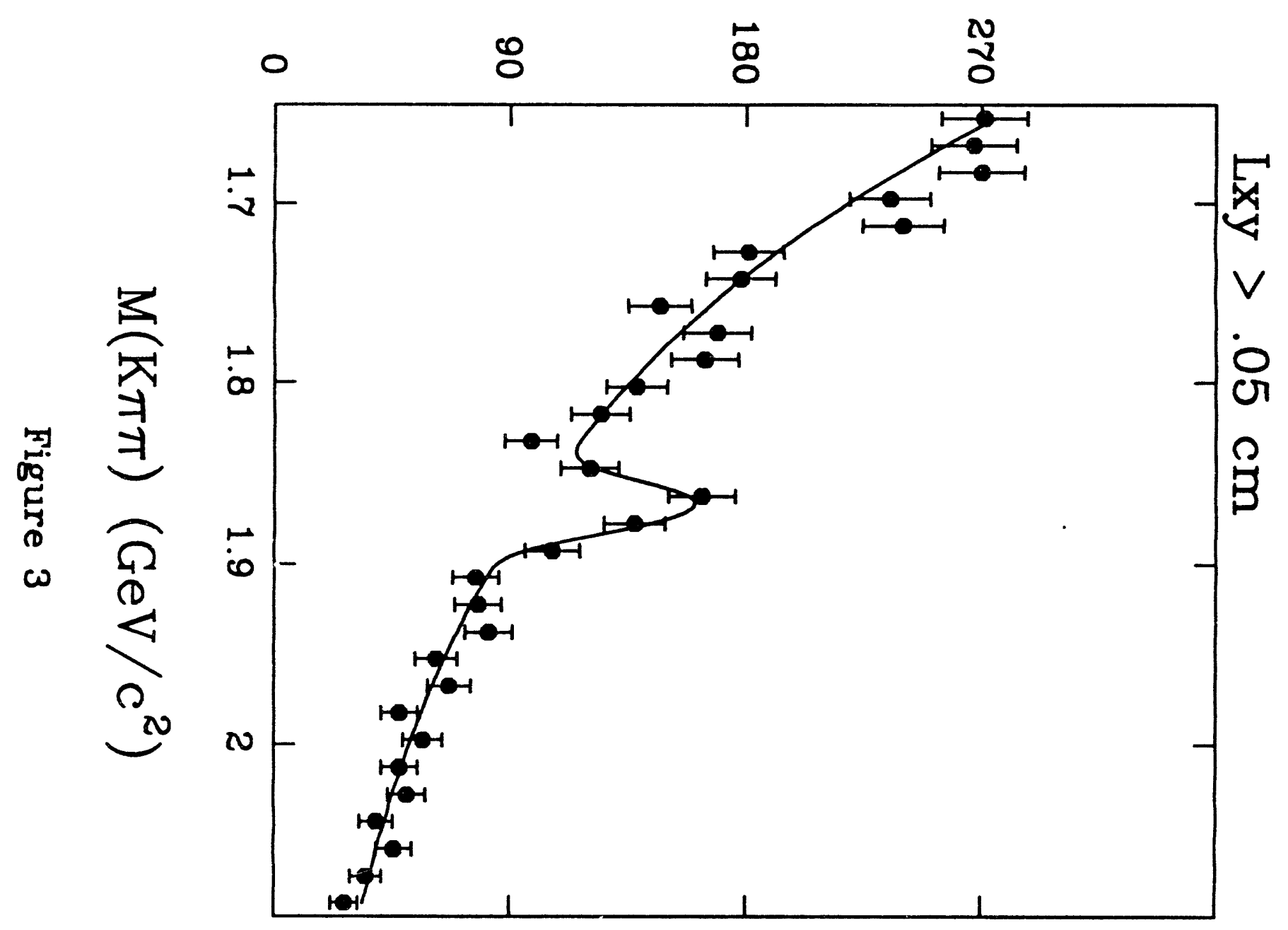


Events/15 MeV

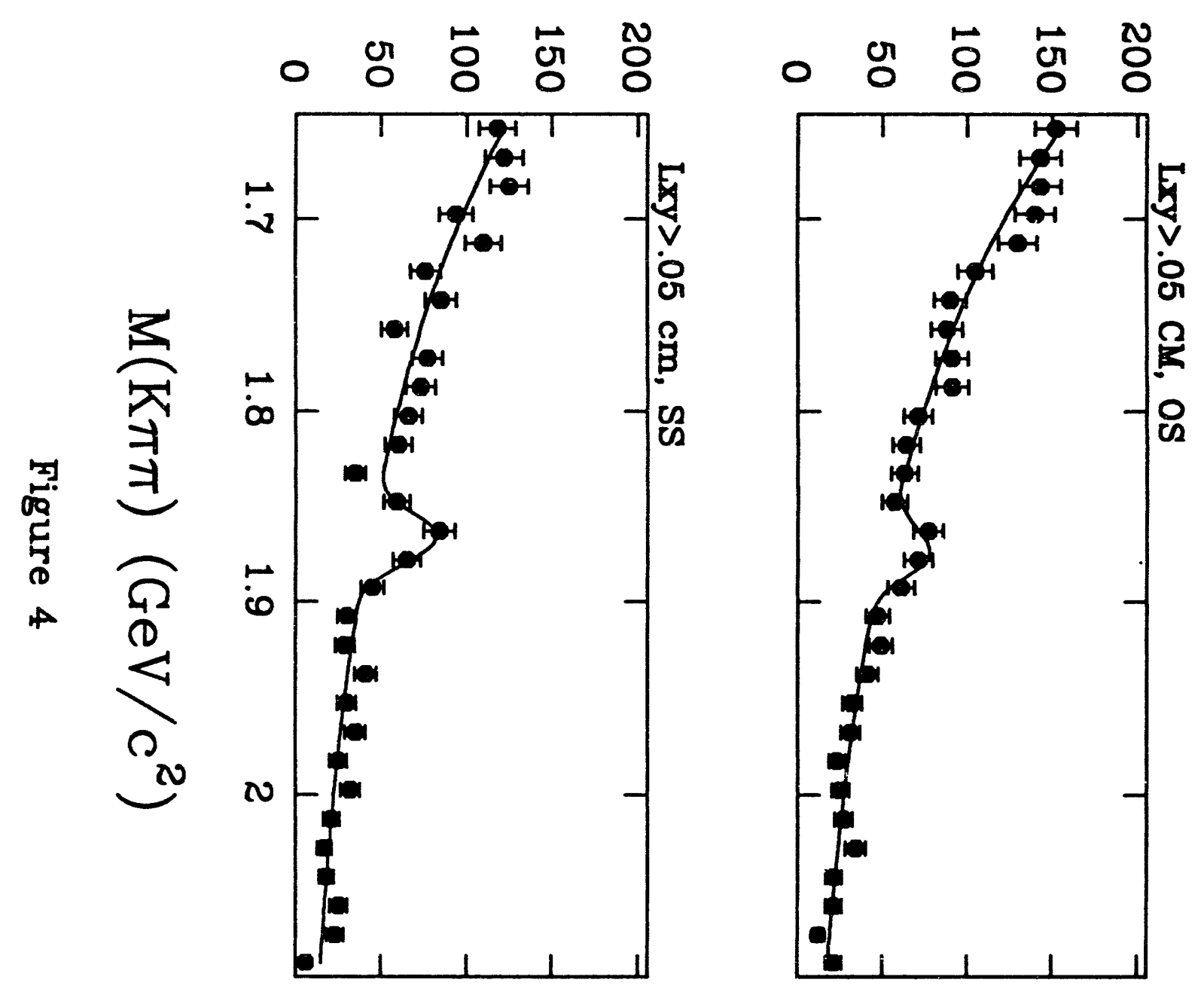




\section{Events/15 MeV}

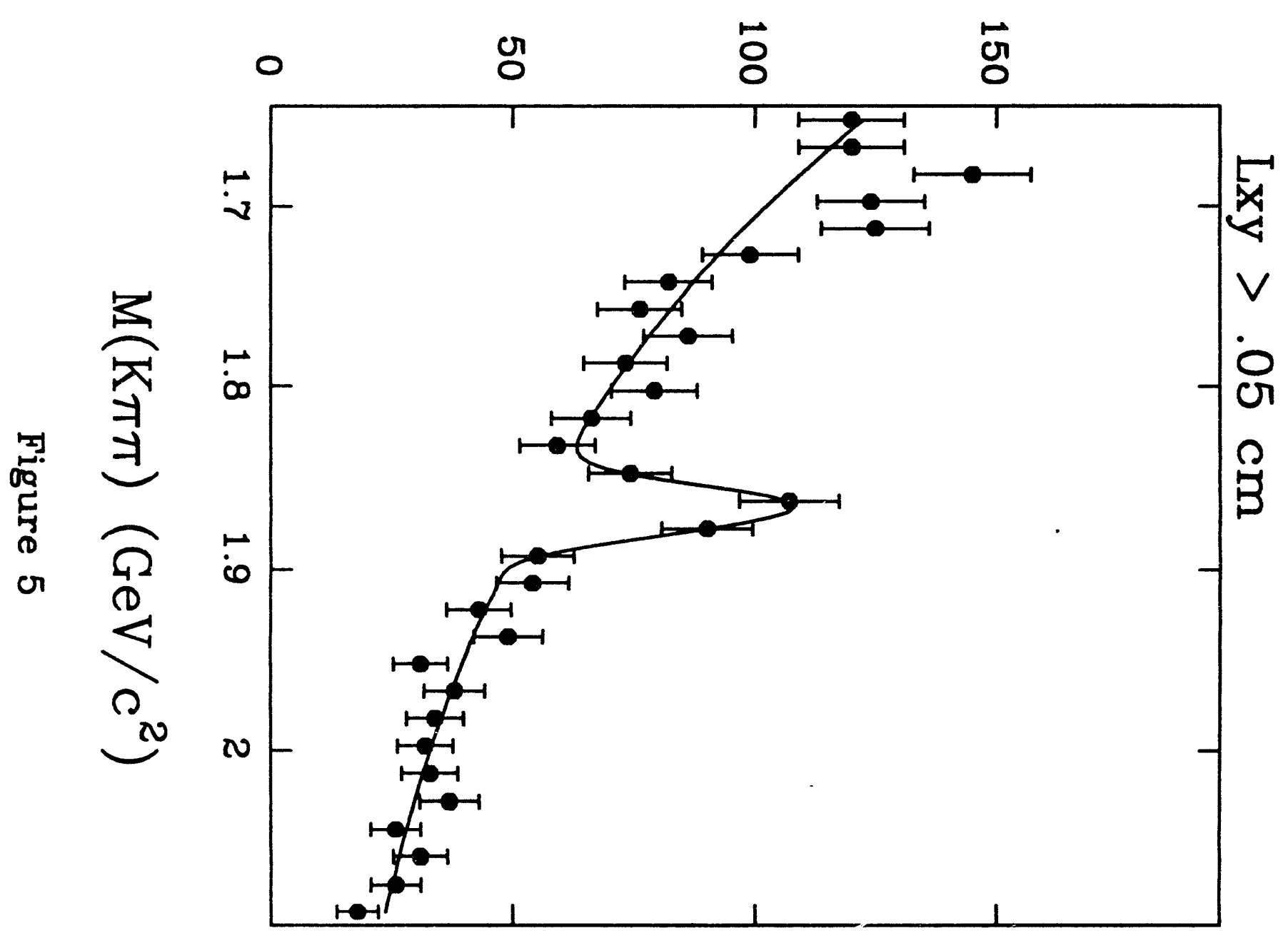




\section{Events/15 MeV}

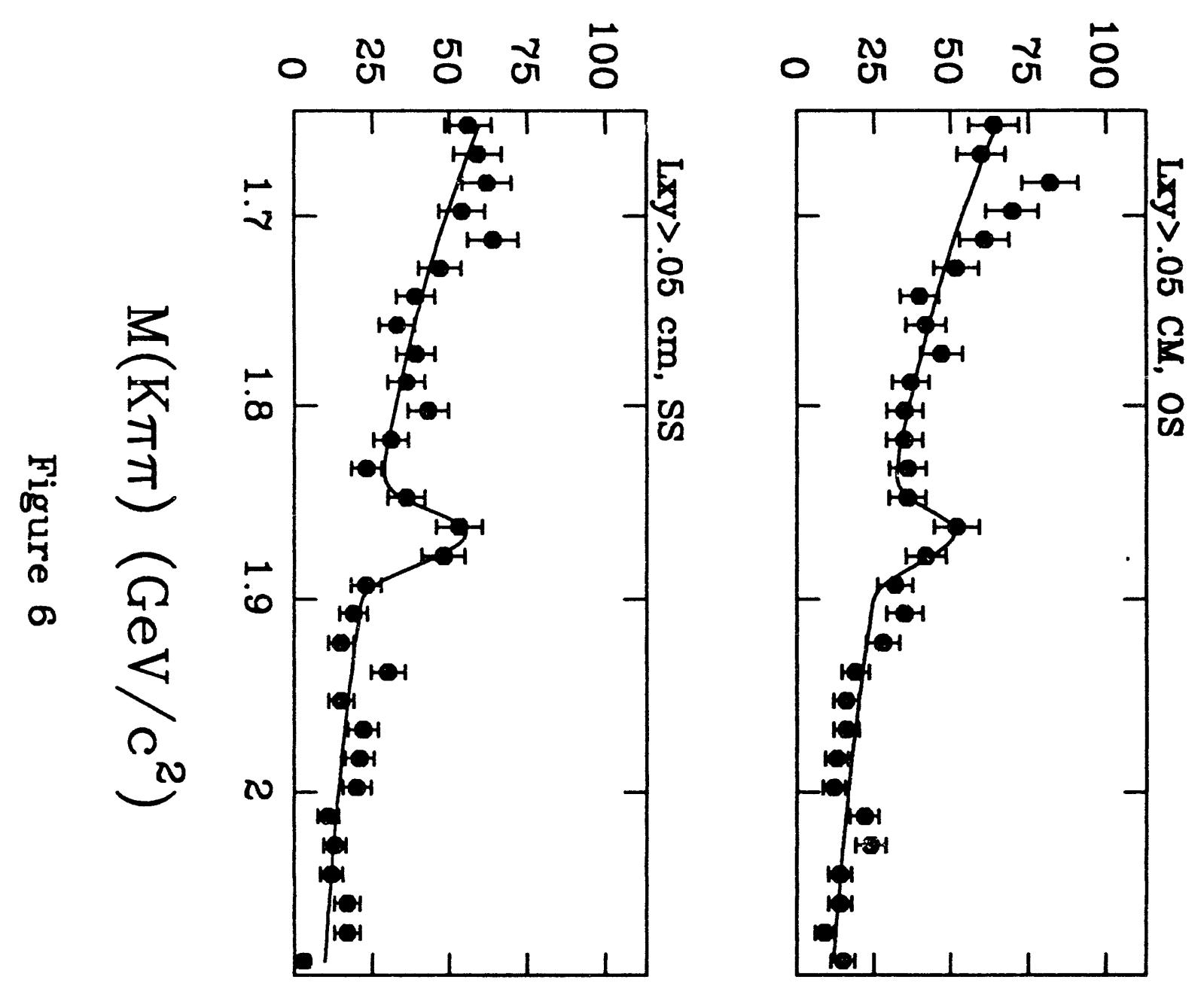




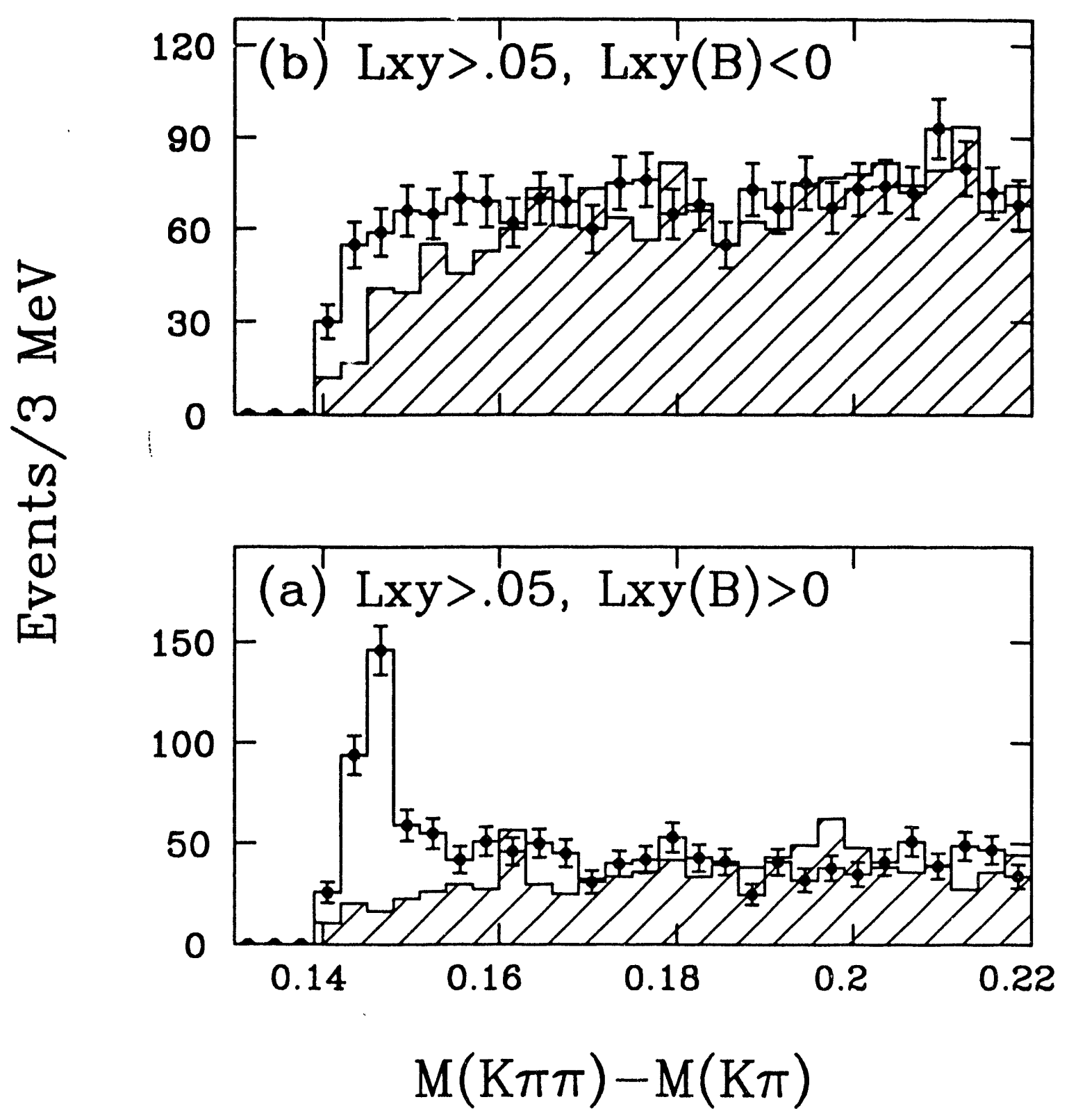

Figure 7 
Events/3 MeV

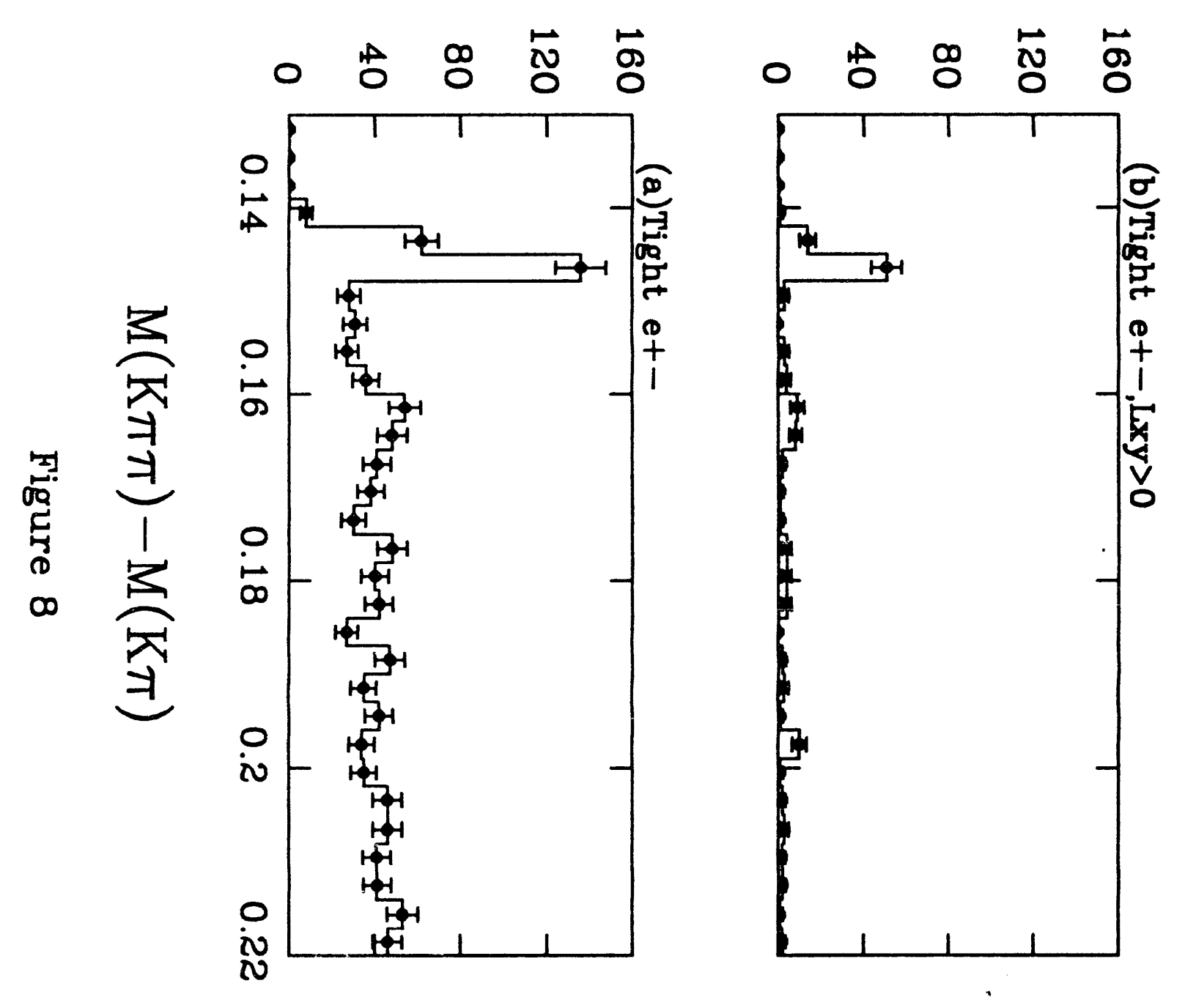




\section{Events/30 MeV}

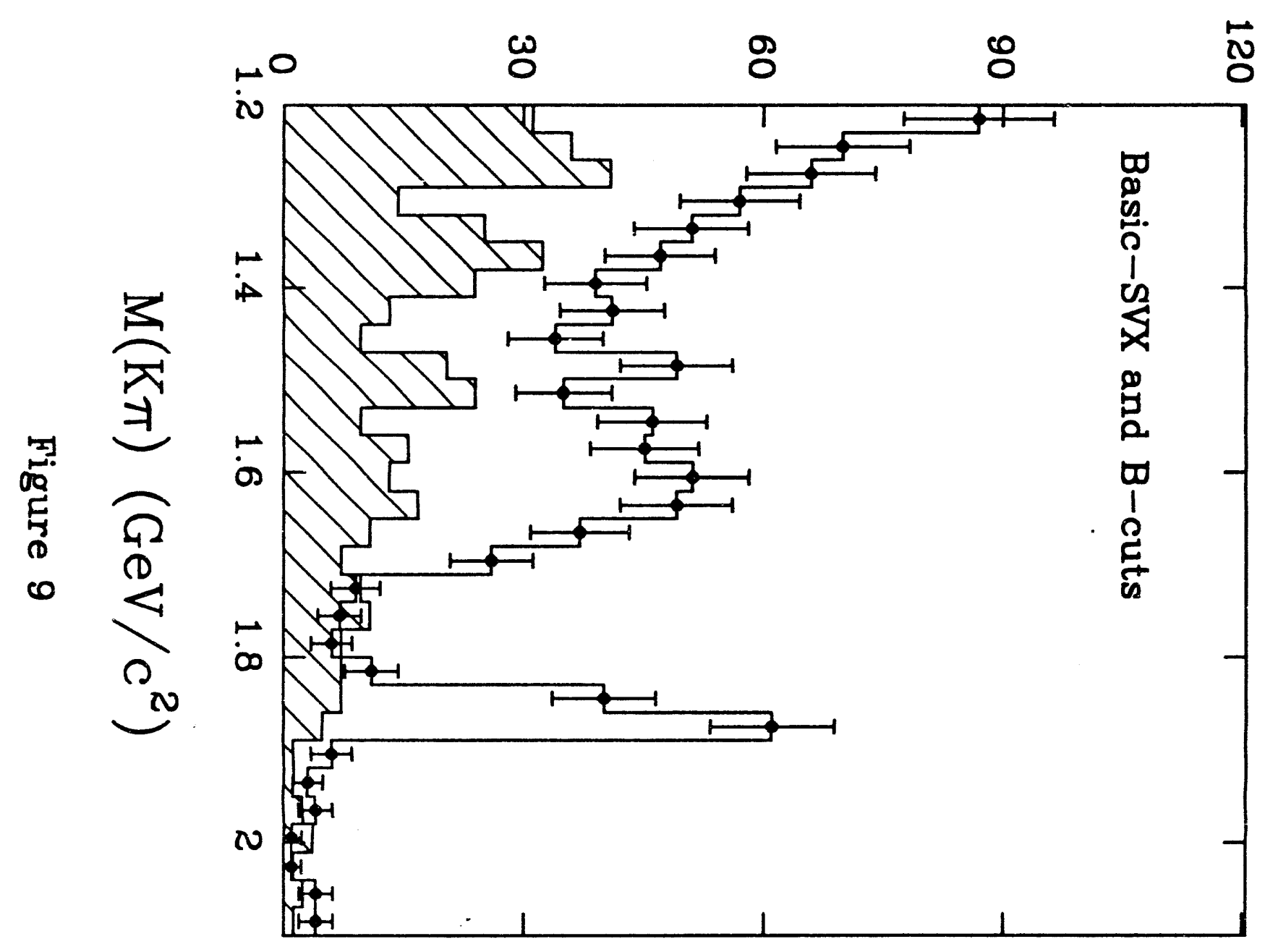




\section{Events/3 MeV}

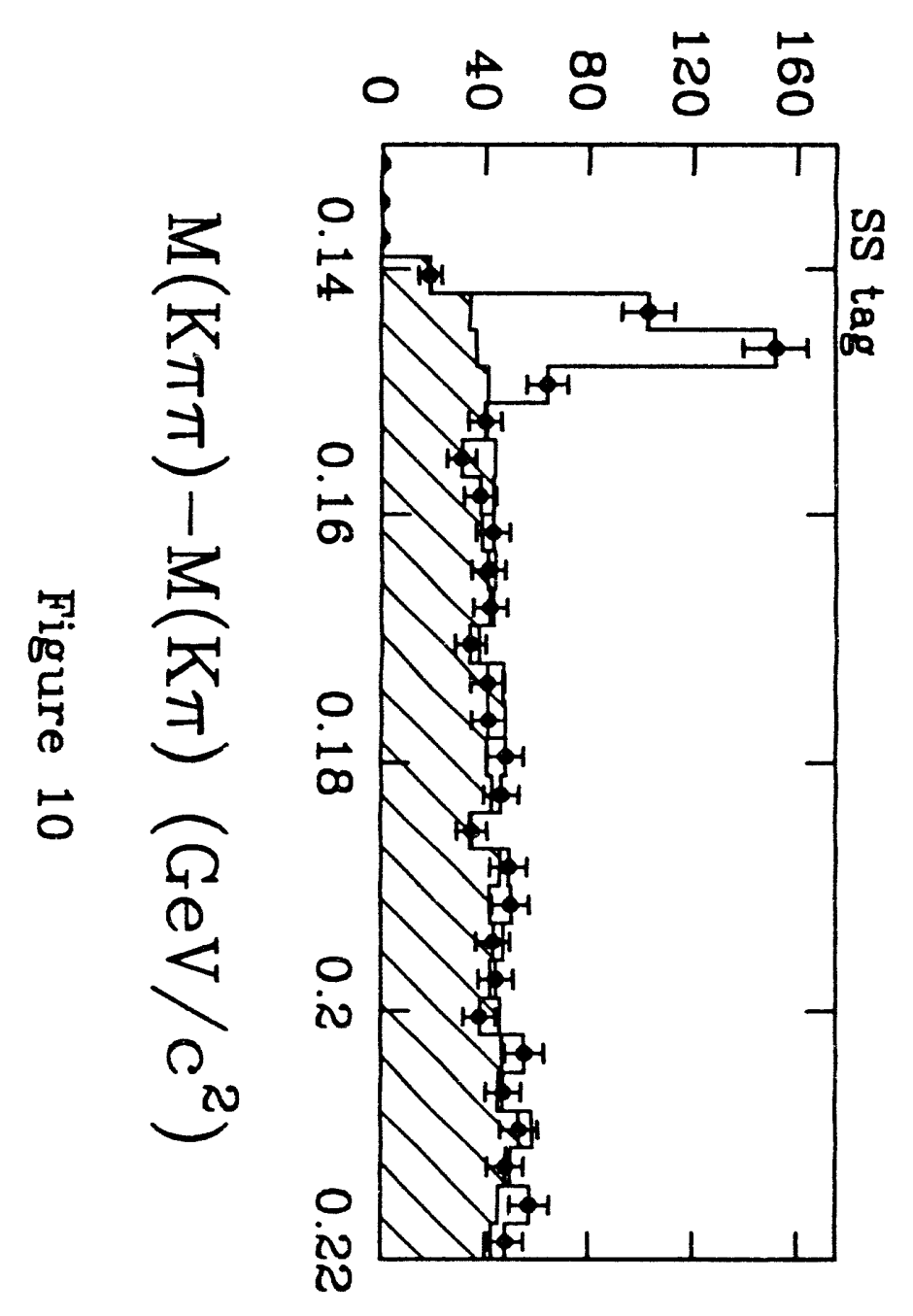

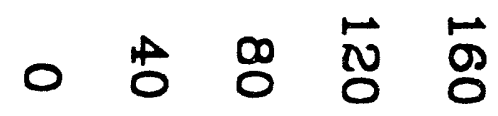

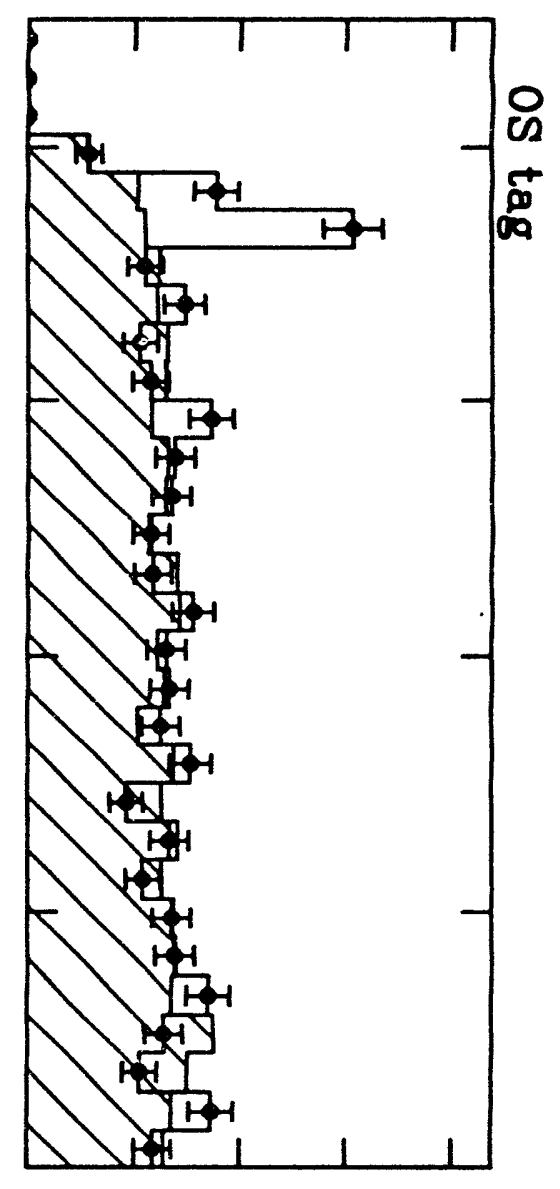




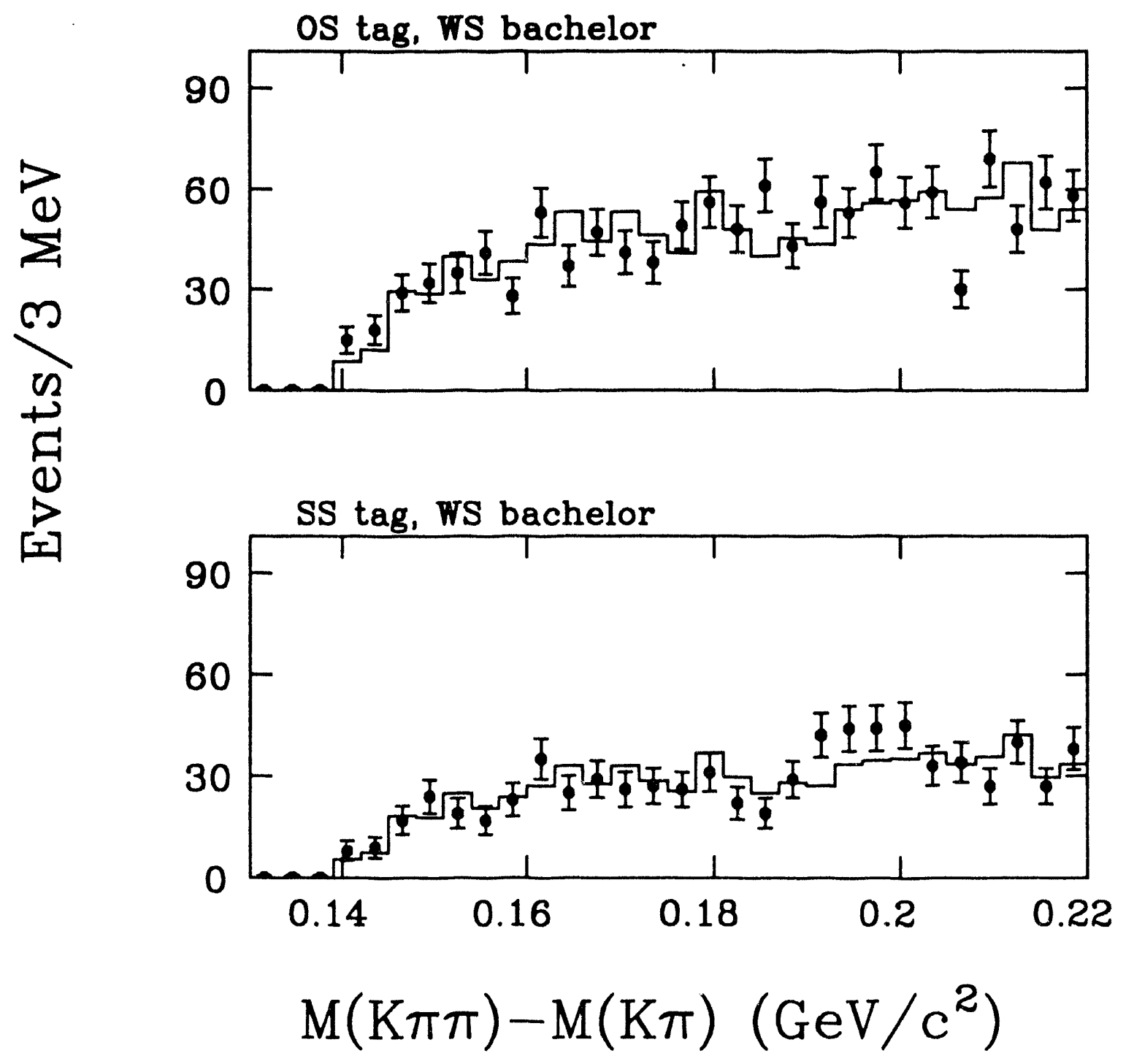

Figure 11 
Events $/ 40 \mu \mathrm{M}$
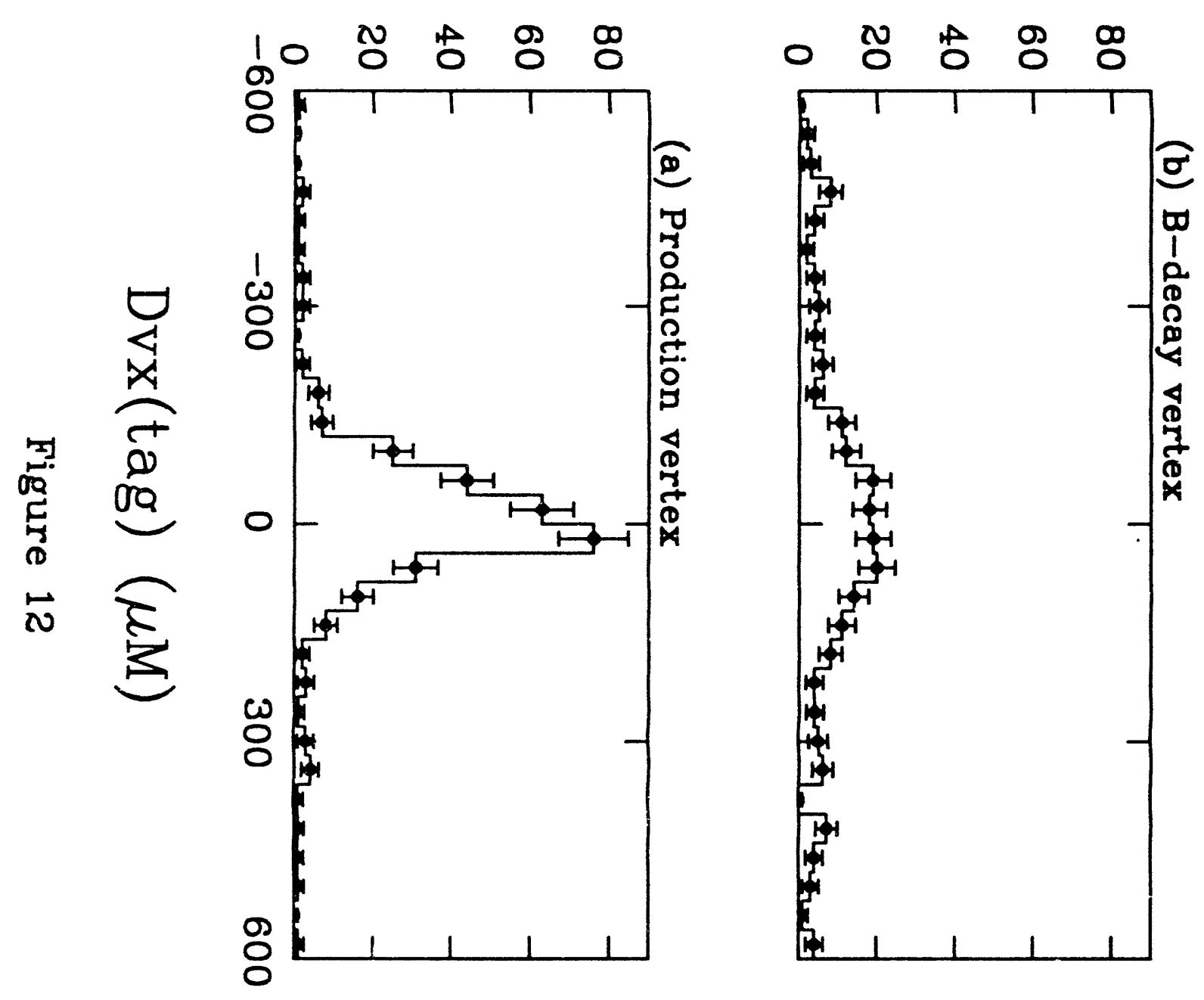
Events $/ 40 \mu \mathrm{M}$

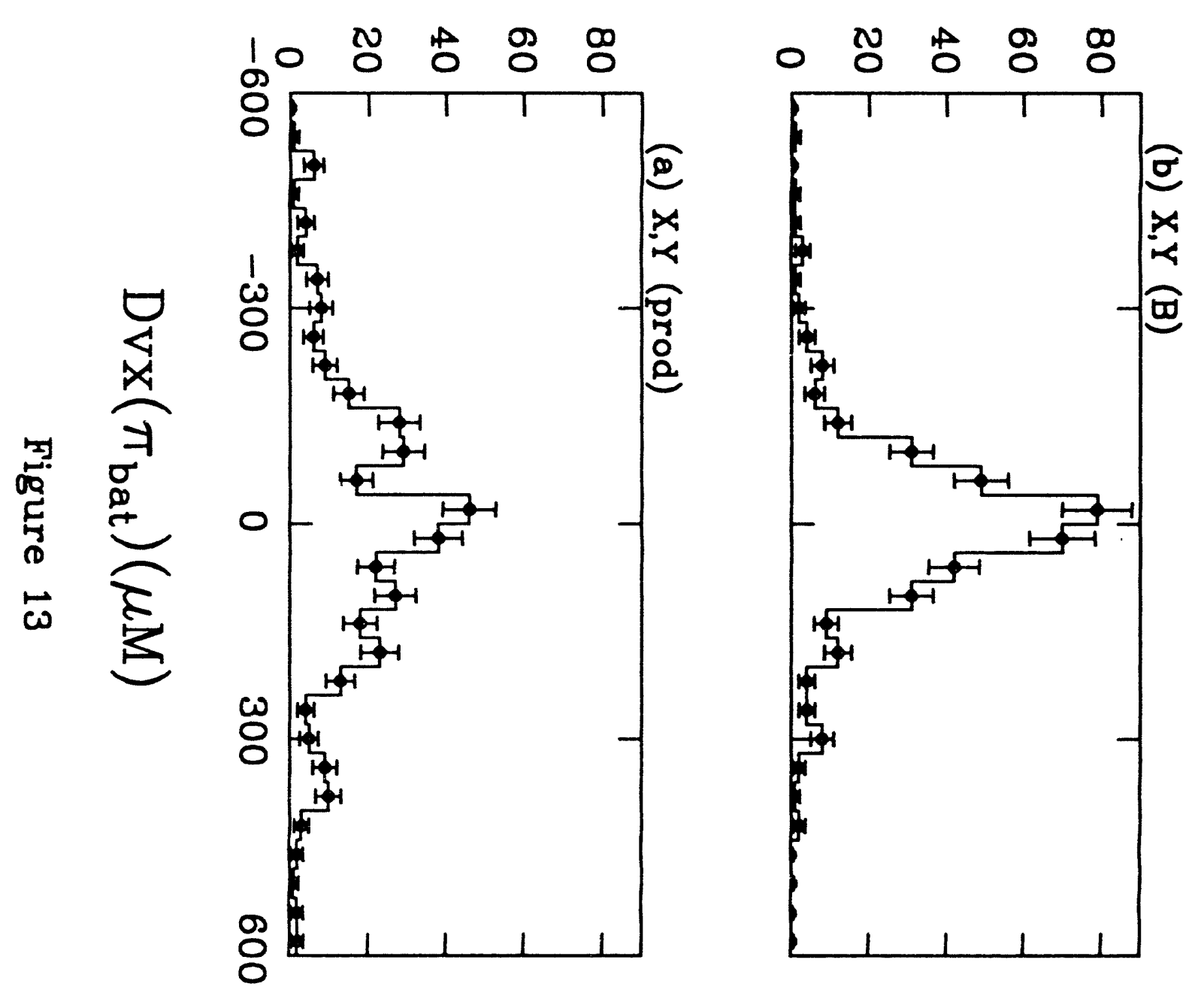



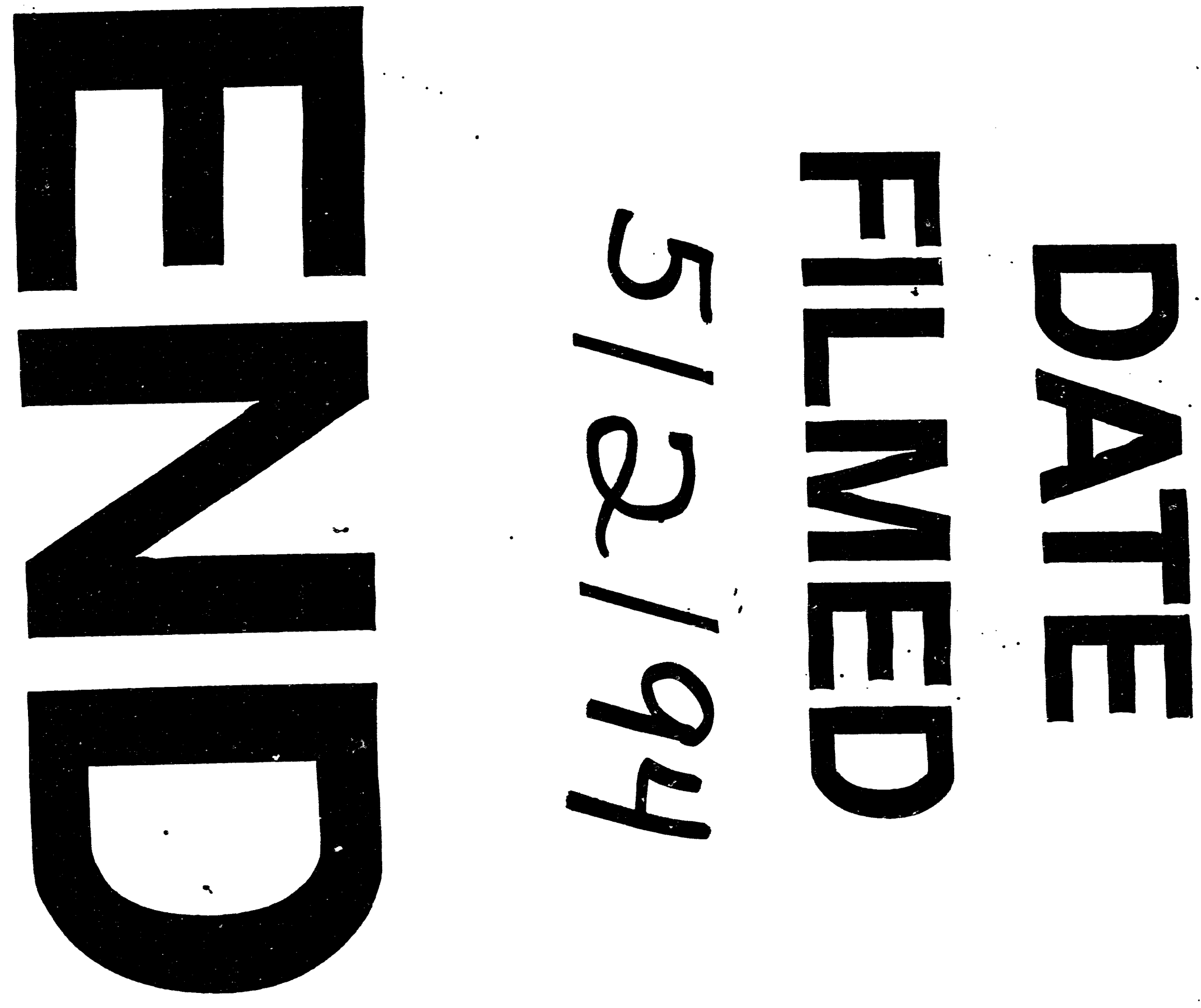
